\title{
Atuação do enfermeiro em situações de desastres naturais: uma revisão integrativa
}

\author{
Nurses' performance in natural disasters situations: an integrative review \\ Desempeño de las enfermeras en situaciones de desastres naturales: una revisión integradora
}

Laine da Costa Almeida Barboza

ORCID: https://orcid.org/0000-0002-9801-1546 Centro Universitário de Ciências e Tecnologia do Maranhão, Brasil E-mail: laynnealmeidah@gmail.com

Karine Costa Melo

ORCID: https://orcid.org/0000-0001-8253-859X

Universidade Federal do Maranhão, Brasil E-mail: karinemelo09@gmail.com

Marconny Lira da Silva

ORCID: https://orcid.org/0000-0003-0334-9294

Centro Tecnológico de Ensino Múltiplo, Brasil E-mail: marconnylirads@hotmail.com

Jaqueline Brito da Costa

ORCID: https://orcid.org/0000-0002-2570-1714

Centro Universitário de Ciências e Tecnologia do Maranhão, Brasil

E-mail: Jaquelinebritto2012@gmail.com

Rogério Cruz Mendes

ORCID: https://orcid.org/0000-0002-9339-6133

Centro Universitário de Ciências e Tecnologia do Maranhão, Brasil

E-mail: rogeriocruz82@yahoo.com

Derik Mikkel de Sousa Silva

ORCID: https://orcid.org/0000-0002-5461-4089

Centro Universitário Maurício de Nassau, Brasil E-mail: derik65mikkel@gmail.com

Victoria Ribeiro de Sousa Marques

ORCID: https://orcid.org/0000-0002-6512-1212

Centro Universitário de Ciências e Tecnologia do Maranhão, Brasil

E-mail: marquesvick24@gmail.com

Paulo Sérgio Gaspar dos Santos

ORCID: https://orcid.org/0000-0003-1763-642X

Centro Universitário de Ciências e Tecnologia do Maranhão, Brasil

E-mail: institutogasph@gmail.com

Linccon Fricks Hernandes

ORCID: https://orcid.org/0000-0002-7642-3080 Escola Superior de Ciências da Santa Casa de Misericórdia de Vitória, Brasil

E-mail: fricksjr@hotmail.com

Ana Carla Marques da Costa

ORCID: https://orcid.org/0000-0002-4246-145X

Centro Universitário de Ciências e Tecnologia do Maranhão, Brasil E-mail: carlama271@gmail.com

Márcia Sousa Santos

ORCID: https://orcid.org/0000-0002-6517-0479

Centro Universitário de Ciências e Tecnologia do Maranhão, Brasil

E-mail: mssenfermeira@gmail.com

Camilla Lohanny Azevedo Viana

ORCID: https://orcid.org/0000-0002-4529-3607

Centro Universitário de Ciências e Tecnologia do Maranhão, Brasil

E-mail: camillalohanny@hotmail.com

Francisléia Falcão França Santos Siqueira

ORCID: https://orcid.org/0000-0001-8783-5139

Universidade Estadual do Maranhão, Brasil E-mail: gpmsaude@gmail.com

Hádila Giovanna Santos Siqueira Cunha

ORCID: https://orcid.org/0000-0002-8401-6119

Universidade Federal do Maranhão, Brasil

E-mail: hadilagiovanna@ hotmail.com

Solygardia Albuquerque Maciel Teixeira

ORCID: https://orcid.org/0000-0003-0214-0982

Centro Universitário Nossa Senhora do Patrocínio, Brasil

E-mail: solygardia@bol.com.br 


\title{
Ana Valéria Lopes Lemos \\ ORCID: https://orcid.org/0000-0002-8708-298X Centro Universitário de Ciências e Tecnologia do Maranhão, Brasil E-mail: anavaleria.lemos@gmail.com
}

\begin{abstract}
Resumo
Os desastres podem acontecer por diversos fenômenos naturais, as vezes pela junção de dois ou mais, sendo classificados em tecnológicos ou naturais. Estes eventos adversos geralmente atingem pessoas que já se encontram em situação de vulnerabilidade, podendo estar relacionada diretamente a transtornos psicológicos, podendo ainda, afetar os profissionais. $\mathrm{O}$ trabalho teve como objetivo geral analisar com base na literatura científica a atuação do enfermeiro em situações de desastres naturais. Trata-se de uma pesquisa bibliográfica do tipo Revisão Integrativa (RI) da literatura, que utilizou os Descritores em Ciências da Saúde (DeCS) e dos títulos da Cumulative Index to Nursing and Allied Health Literature (CINAHL) e Mesh terms. Ao todo foram selecionados onze estudos, a maior parte apresentou abordagem qualitativa dos dados, correspondendo a sete $(63,3 \%)$ trabalhos, em que a base de dados da National Library of Medicine apresentou o maior número de estudos $(45,4 \%)$. A maior parte das publicações concentraram-se no ano de 2019 (36,3\%) e 2020 (27,2\%). Quanto ao idioma dez (90\%) das publicações estavam na língua inglesa; em que o Brasil foi o país com o maior número de publicações $(27,2 \%)$. A assistência prestada aos pacientes vítimas de desastres naturais, deve ser focada em aspectos físicos e psicológicos. Nesse caso, a enfermagem deve entrar em ação lidando diretamente com o sujeito e prestando esclarecimentos acerca do procedimento realizado por ele, além de realizar escuta ativa, quando o paciente tem condições e deseja verbalizar, oferecer conforto e utilizar o toque como fonte de segurança aos clientes.
\end{abstract}

Palavras-chave: Enfermagem; Serviços médicos de emergência; Administração de desastres; Desastres naturais.

\begin{abstract}
Disasters can happen due to several natural phenomena, sometimes by the joining of two or more, being classified as technological or natural. These adverse events usually affect people who are already in a vulnerable situation, and may be directly related to psychological disorders, and may also affect professionals. The work aimed to analyze, based on the scientific literature, the role of nurses in situations of natural disasters. This is an Integrative Review (IR) literature search of the literature, which used the Descriptors in Health Sciences (DeCS) and the titles of the Cumulative Index to Nursing and Allied Health Literature (CINAHL) and Mesh terms. In all, eleven studies were selected, most of which presented a qualitative approach to data, corresponding to seven $(63.3 \%)$ works, in which the National Library of Medicine database had the largest number of studies (45.4\%). Most publications focused on the year $2019(36.3 \%)$ and $2020(27.2 \%)$. As for the language, ten (90\%) of the publications were in English; where Brazil was the country with the highest number of publications $(27.2 \%)$. The assistance provided to patients victims of natural disasters should be focused on physical and psychological aspects. In this case, nursing must take action by dealing directly with the subject and providing clarifications about the procedure performed by him, in addition to performing active listening, when the patient is able and willing to verbalize, offer comfort and use touch as a source of security for customers.
\end{abstract}

Keywords: Nursing; Emergency medical services; Disaster management; Natural disasters.

\section{Resumen}

Los desastres pueden ocurrir debido a varios fenómenos naturales, a veces por la unión de dos o más, siendo clasificados como tecnológicos o naturales. Estos eventos adversos suelen afectar a personas que ya se encuentran en una situación de vulnerabilidad, y pueden estar directamente relacionados con trastornos psicológicos, pudiendo afectar también a los profesionales. El trabajo tuvo como objetivo analizar, con base en la literatura científica, el papel de las enfermeras en situaciones de desastres naturales. Esta es una búsqueda bibliográfica de la Revisión Integrativa (IR) de la literatura, que utilizó los Descriptores en Ciencias de la Salud (DeCS) y los títulos del Índice acumulativo de literatura de enfermería y salud afín (CINAHL) y los términos Mesh. En total, se seleccionaron once estudios, la mayoría de los cuales presentaban un abordaje cualitativo de los datos, correspondientes a siete $(63,3 \%)$ trabajos, en los que la base de datos de la Biblioteca Nacional de Medicina tenía el mayor número de estudios (45,4\%). La mayoría de las publicaciones se centraron en el año 2019 (36,3\%) y 2020 (27,2\%). En cuanto al idioma, diez (90\%) de las publicaciones fueron en inglés; donde Brasil fue el país con mayor número de publicaciones (27,2\%). La asistencia brindada a los pacientes víctimas de desastres naturales debe centrarse en los aspectos físicos y psicológicos. En este caso, la enfermería debe actuar tratando directamente con el sujeto y proporcionando aclaraciones sobre el procedimiento que realiza, además de realizar una escucha activa, cuando el paciente es capaz y está dispuesto a verbalizar, ofrecer comodidad y utilizar el tacto como fuente de seguridad para los clientes.

Palabras clave: Enfermería; Servicios médicos de emergencia; Gestión de desastres; Desastres naturales. 


\section{Introdução}

Os desastres podem acontecer por diversos fenômenos naturais, as vezes pela junção de dois ou mais, sendo classificados em tecnológicos ou naturais (Guha-Sapir et al., 2015). Os desastres tecnológicos são aqueles que ocorrem devido a uma intenção humana, negligência ou erro de um sistema humano, o que resulta em significativos danos ou mortes (Zhouri et al., 2016). Já os desastres naturais são ocasionados pelo impacto de um perigo natural em um local socioeconômico que apresenta certa vulnerabilidade (Moss et al., 2018).

A ocorrência de desastres naturais se relaciona a diversos fatores e ocasiona diversas consequências tanto físicas quanto psicológicas. Neste aspecto, destaca-se que a estes eventos adversos geralmente atingem pessoas que já se encontram em situação de vulnerabilidade, agravando a condição financeira destes indivíduos e se relacionando diretamente ao aparecimento de transtornos psicológicos como o estresse pós-traumático após a vivência de catástrofes, podendo ainda, afetar os profissionais (Cohen et al., 2019).

Nessa perspectiva observa-se que, a atuação dos profissionais de enfermagem vai além da assistência hospitalar, onde os mesmos devem ser capacitados para fornecer educação, de forma a incitar a conscientização sobre prevenção de desastres, provisão e educação sobre respostas a pessoas feridas e doentes e individualidade, propondo apoio psicológico (Hamatate et al., 2019).

Destaca-se a necessidade de fornecer informações confiáveis a profissionais da saúde bem como o público geral. Neste aspecto, a formulação de políticas públicas sobre a prevenção de desastres naturais, com base em evidências científicas, e sua disseminação, poderia promover uma comunicação efetiva. Minimizando as consequências dos desastres e promover o fortalecimento da sociedade como um todo (Jillson et al., 2019).

Diante disso o estudo tem como questão norteadora: Quais evidências científicas mostram a atuação do enfermeiro em situações de desastres naturais? Para tal, o objetivo geral é analisar com base na literatura científica a atuação do enfermeiro em situações de desastres naturais, e os objetivos específicos identificar as estratégias de enfrentamento dos profissionais de enfermagem frente aos desastres naturais; explanar sobre os principais riscos que podem afetar a saúde dos enfermeiros que atuam nos desastres naturais; e descrever o grau de preparação dos profissionais de Saúde para atuar frente a situações de desastres.

Devido à grande elevação de situações de desastres naturais com múltiplas vítimas em esfera mundial, faz-se necessário a realização de estudos que abordem alguns aspectos relacionados à prestação dos serviços do enfermeiro que é uma peça fundamental diante das situações de calamidade pública. Torna-se claro a necessidade de avaliar a atuação do enfermeiro no atendimento pré-hospitalar em situações de desastres naturais. Dando ênfase aos principais serviços prestados; identificando os principais impactos causados pelos desastres naturais na vida dos profissionais atuantes, detalhando o tipo de qualificação que os enfermeiros recebem para lidar com vítimas de desastres naturais.

\section{Metodologia}

O presente estudo trata-se de uma pesquisa bibliográfica do tipo Revisão Integrativa (RI) da literatura. Este procedimento foi escolhido por possibilitar a síntese e análise do conhecimento científico já produzido sobre o tema “Atuação do enfermeiro em situações de desastres naturais: uma revisão integrativa". Esta revisão utilizou a metodologia proposta no estudo de Oliveira et al. (2016).

Considerando a necessidade de garantir o cuidado com base na Prática Baseada em Evidências (PBE), a RI torna-se um instrumento válido para a PBE no cenário atual da enfermagem. A RI é uma ferramenta que sintetiza as investigações sobre determinado assunto, de modo que, direciona a prática embasada no conhecimento cientifico. Este tipo de estudo é complexo e desafiador, no entanto, uma abordagem sistemática e rigorosa, na busca das evidências e análise dos dados, resulta em vieses e 
erros reduzidos, trazendo dados mais fidedignos (Souza et al., 2010).

A RI está estruturada em seis fases, sendo elas: Identificação do tema e seleção da hipótese ou questão de pesquisa; Estabelecimento de critérios para inclusão e exclusão; Identificação dos estudos pré-selecionados e selecionados; Avaliação dos estudos incluídos; Intepretação dos resultados; e Apresentação da revisão/síntese do conhecimento (Sousa et al., 2017)

O tema "Atuação do enfermeiro em situações de desastres naturais: uma revisão integrativa", determinou a construção da estratégia PICO, que representa um acrônimo para Paciente (P), Intervenção (I), Comparação (C) e Desfechos (Ooutcomes), na qual foi utilizada para a geração da questão norteadora desta revisão integrativa da literatura: "Quais evidências científicas mostram a atuação do enfermeiro em situações de desastres naturais?"

Para a localização dos estudos relevantes, que respondessem à pergunta de pesquisa, utilizou-se de descritores indexados e não indexados (palavras-chave) nos idiomas português, inglês e espanhol. Os descritores foram obtidos a partir do Medical Subject Headings (MESH), dos Descritores em Ciências da Saúde (DeCS) e dos títulos da Cumulative Index to Nursing and Allied Health Literature (CINAHL), conforme demonstrado no Quadro 01.

Consultou-se por meio de descritores e palavras-chave as bases de dados PubMed da National Library of Medicine; Biblioteca Virtual da Saúde (BVS), coordenada pela BIREME e composta de bases de dados bibliográficas produzidas pela Rede BVS, como a Literatura Latino-Americana e do Caribe em Ciências da Saúde (LILACS), além da base de dados Medline e outros tipos de fontes de informação; e CINAHL.

Quadro 1. Elementos da estratégia PICO e descritores utilizados. Caxias, MA, Brasil, 2021.

\begin{tabular}{|c|c|c|c|c|}
\hline \multicolumn{2}{|r|}{ Elementos } & MESH & DECS & Títulos CINAHL \\
\hline $\mathrm{P}$ & Enfermeiro & "Nursing" & $\begin{array}{c}\text { "Enfermagem" } \\
\text { "Nursing" } \\
\text { "Enfermería" }\end{array}$ & "Nursing" \\
\hline I & $\begin{array}{l}\text { Atendimento de } \\
\text { saúde }\end{array}$ & - & $\begin{array}{l}\text { "Serviços Médicos de Emergência" } \\
\text { "Emergency Medical Services" } \\
\text { "Servicios Médicos de Urgencia" } \\
\text { "Administração de Desastres" } \\
\text { "Disaster Management" } \\
\text { "Administración de Desastres" }\end{array}$ & $\begin{array}{c}\text { "Emergency Medical Services" } \\
\text { "Disaster Management" }\end{array}$ \\
\hline $\mathrm{C}$ & - & - & - & - \\
\hline $\mathrm{O}$ & Desastres naturais & "Natural Disasters" & $\begin{array}{l}\text { "Desastres Naturais" } \\
\text { "Natural Disasters" } \\
\text { "Desastres Naturales" }\end{array}$ & "Natural Disasters" \\
\hline
\end{tabular}

Fonte: Descritores e Títulos (2021).

O elemento $\mathrm{C}$ da estratégia PICO não foi abordado nesta pesquisa pois esta não tem por objetivo comparar intervenções. Os termos utilizados durante a pesquisa foram classificados e combinados nos bancos de dados, resultando em estratégias específicas de cada base (Quadro 2). 
Quadro 2. Estratégias de busca utilizadas nas bases de dados BIREME, PUBMED e CINAHL. Caxias, MA, Brasil, 2021.

\begin{tabular}{|c|c|c|c|c|}
\hline BASE DE DADOS & ESTRATÉGIA DE BUSCA & 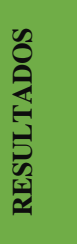 & 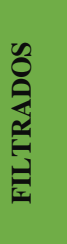 & 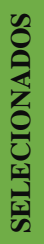 \\
\hline $\begin{array}{c}\text { BIREME } \\
\text { (descritores Decs) }\end{array}$ & $\begin{array}{l}\text { (Nursing) AND (Emergency Medical Services) OR (Disaster Management) AND } \\
\text { (Natural Disasters) }\end{array}$ & 99 & 26 & 02 \\
\hline $\begin{array}{l}\text { PubMed } \\
\text { (descriptors MeSH) }\end{array}$ & (Nursing) AND (Natural Disasters) & 731 & 222 & 05 \\
\hline $\begin{array}{l}\text { CINAHL } \\
\text { (CINAHL } \\
\text { Headings) }\end{array}$ & $\begin{array}{l}\text { Nursing AND Emergency Medical Services OR Disaster Management AND Natural } \\
\text { Disasters }\end{array}$ & 3930 & 145 & 04 \\
\hline
\end{tabular}

Fonte: Bases de dados (2021).

Como critérios de inclusão utilizaram-se estudos disponíveis em sua totalidade, publicados nos últimos cinco anos, de 2016 até 2021, nos idiomas Português, Espanhol e Inglês. Foram excluídos da busca inicial capítulos de livros, resumos, textos incompletos, teses, dissertações, monografias, relatos técnicos e outras formas de publicação que não fossem artigos científicos completos.

A análise para seleção dos estudos foi realizada em duas fases, a saber: Na primeira, os estudos foram préselecionados segundo os critérios de inclusão e exclusão e de acordo com a estratégia de funcionamento e busca de cada base de dados.

Encontrou-se noventa e nove (99) estudos como busca geral na BVS, sendo que limitando a busca para artigos com texto completo realizado com humanos nos últimos cinco anos, obteve-se vinte e seis (26) estudos, destes foram analisados títulos e resumos onde apenas dois (02) estudos foram condizentes com a questão desta pesquisa.

Na base PUBMED, como busca total foram encontrados setecentos e trinta e um (731) estudos, aplicando na pesquisa o filtro que limita por texto completo dos últimos cinco anos com humanos, obteve-se duzentos e vinte e dois (222) estudos, destes foram analisados títulos e resumos e teve como resultado final de cinco (5) estudos.

Na CINAHL foram obtidos três mil e novecentos e trinta (3930) estudos como busca geral, sendo que limitando a busca para artigos com texto completo realizado nos últimos cinco anos com humanos, obteve-se cento e quarenta e cinco (145) estudos, sendo quatro (4) foram condizentes com a questão desta pesquisa após a análise dos títulos e resumos.

$\mathrm{Na}$ segunda fase os estudos foram analisados quanto ao potencial de participação no estudo, avaliando o atendimento à questão de pesquisa, bem como o tipo de investigação, objetivos, amostra, método, desfechos, resultados e conclusão, resultando em onze (11) artigos.

As informações coletadas nos artigos científicos foram organizadas em categorias analíticas que facilitou a ordenação e a sumarização de cada estudo. Essa categorização foi realizada de forma descritiva, indicando os dados mais relevantes para o estudo.

A pesquisa levou em consideração os aspectos éticos da pesquisa quanto às citações dos estudos, respeitando a autoria das ideias, os conceitos e as definições presentes nos artigos incluídos na revisão. Optou-se pela análise em forma estatística e de forma de texto, utilizando cálculos matemáticos e inferências, que foram apresentados em quadros e tabelas para facilitar a visualização e compreensão.

As evidências científicas foram classificadas segundo os níveis e graus de recomendação propostos por Bork (2011): Nível 1- Revisão sistemática; 2- Ensaio Clínico Randomizado; 3- Estudo de Coorte; 4- Caso e controle; 5- Séries de casos; 6Opinião de especialista; 7- Estudos pré-clínicos/ in vitro. E quanto ao grau de recomendação: A- Resultado recomenda a 
intervenção; B- Resultado não é conclusivo - não é suficiente para confirmar a hipótese; C- Resultado contraindica a intervenção.

\section{Resultados}

Essa fase é organizada em duas partes. A primeira está relacionada com a caracterização dos estudos, já a segunda, relaciona-se ao cumprimento do objetivo do estudo, que consiste em conhecer a atuação do enfermeiro em situações de desastres naturais.

Dos 11 artigos incluídos nesta revisão, a maior parte apresentou abordagem qualitativa dos dados, correspondendo a sete $(63,3 \%)$ trabalhos, em que a base de dados da National Library of Medicine apresentou o maior número de estudos (45,4\%). A maior parte das publicações concentraram-se no ano de 2019 (36,3\%) e 2020 (27,2\%). Quanto ao idioma dez (90\%) das publicações estavam na língua inglesa; em que o Brasil foi o país com o maior número de publicações (27,2\%).

O periódico com maior percentual de ocorrência foi Prehospital and Disaster Medicine $(18,1 \%)$. Com relação ao delineamento de pesquisa houve predomínio de estudos transversais $(63,6 \%)$; seguido de revisões sistemática da literatura (27,2\%). O nível de evidencia predominante foi seis (63,6\%), seguido de um $(27,2 \%)$ e cinco $(9,0 \%)$, respectivamente. Onde cerca de $72,7 \%$ dos estudos obtiveram grau de recomendação "A” para a prática clínica, conforme demonstrado na Tabela 1. 
Tabela 1. Análise descritiva das produções científicas acerca da atuação do enfermeiro em situações de desastres naturais. Caxias, MA, Brasil, 2021. ( $\mathrm{N}=11)$.

\begin{tabular}{|c|c|c|}
\hline Variáveis & $\mathbf{N}$ & $\%$ \\
\hline \multicolumn{3}{|l|}{ Base de dados } \\
\hline PUBMED & 05 & 45,4 \\
\hline CINAHL & 04 & 36,3 \\
\hline BIREME & 02 & 18,1 \\
\hline \multicolumn{3}{|l|}{ Abordagem do estudo } \\
\hline Quantitativo & 04 & 36,3 \\
\hline Qualitativo & 07 & 63,6 \\
\hline \multicolumn{3}{|l|}{ Ano } \\
\hline 2017 & 02 & 18,1 \\
\hline 2018 & 01 & 09,0 \\
\hline 2019 & 04 & 36,3 \\
\hline 2020 & 03 & 27,2 \\
\hline 2021 & 01 & 09,0 \\
\hline \multicolumn{3}{|l|}{ Idiomas } \\
\hline Inglês & 10 & 90,0 \\
\hline Português & 01 & 09,0 \\
\hline \multicolumn{3}{|l|}{ País } \\
\hline China & 02 & 18,1 \\
\hline Filipinas & 01 & 09,0 \\
\hline Brasil & 03 & 27,2 \\
\hline Australia & 01 & 09,0 \\
\hline Estados Unidos & 03 & 27,2 \\
\hline Omã & 01 & 09,0 \\
\hline \multicolumn{3}{|l|}{ Periódicos } \\
\hline Journal of Clinical Nursing & 01 & 09,0 \\
\hline International Journal for Human Caring & 01 & 09,0 \\
\hline Journal Of Emergency Nursing & 01 & 09,0 \\
\hline Australasian Emergency Nursing Journal & 01 & 09,0 \\
\hline Nurse Education Today & 01 & 09,0 \\
\hline Journal of Nursing Education & 01 & 09,0 \\
\hline Revista Brasileira de Enfermagem & 01 & 09,0 \\
\hline Prehospital and Disaster Medicine & 02 & 18,1 \\
\hline Rural and Remote Health & 01 & 09,0 \\
\hline International Council of Nurses & 01 & 09,0 \\
\hline \multicolumn{3}{|l|}{ Delineamento de pesquisa } \\
\hline Revisão sistemática da literatura & 03 & 27,2 \\
\hline Estudo de revisão & 01 & 09,0 \\
\hline Estudo transversal & 07 & 63,6 \\
\hline \multicolumn{3}{|l|}{ Classificação da evidência } \\
\hline Nível 01 & 03 & 27,2 \\
\hline Nível 05 & 01 & 09,0 \\
\hline Nível 06 & 07 & 63,6 \\
\hline \multicolumn{3}{|l|}{ Grau de recomendação } \\
\hline A & 08 & 72,7 \\
\hline $\mathrm{B}$ & 03 & 27,2 \\
\hline
\end{tabular}

Fonte: Dados da pesquisa (2021).

O Quadro 3 mostra a distribuição dos estudos segundo autores e anos de publicação, título; tipo de estudo; objetivo principal e perfil amostral. Os estudos versaram sobre as experiências dos profissionais de enfermagem frente a desastres naturais, o conceito de cuidado em desastres naturais também é abordado por teóricos de enfermagem.

Além da experiencia e conceitos, os autores retratam as competências de enfermagem frente aos desastres, em que são demonstradas as intervenções nas situações de emergência em diferentes contextos e nível de atenção, quer sejam na atenção primária, secundária ou terciária de saúde, onde a preparação da equipe de enfermagem frente a estas condições também são abordadas, além dos programas de educação e treinamento em enfermagem em desastres. Esses pontos estão retratados na discussão deste trabalho. 
Quadro 3. Publicações incluídas segundo o título do artigo, autor, objetivo principal e perfil amostral. Caxias, MA, Brasil. 2021. (N=11).

\begin{tabular}{|c|c|c|c|c|}
\hline $\begin{array}{l}\mathbf{N}^{0} \text { de ordem } \\
\text { e Base }\end{array}$ & Título do artigo & Autor/ano & Objetivo principal & Perfil amostral \\
\hline $\begin{array}{c}\text { A1 } \\
\text { BIREME }\end{array}$ & $\begin{array}{l}\text { Experiences of nurses involved in natural } \\
\text { disaster relief: A meta-synthesis of qualitative } \\
\text { literature }\end{array}$ & $\begin{array}{l}\text { Xue, Shu, Hayter e Lee } \\
\text { (2020) }\end{array}$ & $\begin{array}{l}\text { Explorar as experiências dos enfermeiros na resposta a } \\
\text { desastres naturais. }\end{array}$ & $\begin{array}{l}\text { O método sistemático de recuperação, análise e interpretação dos achados de } \\
\text { Sandelowski e Barroso foi usado para produzir um meta-resumo dos achados } \\
\text { de } 10 \text { artigos avaliando experiências em } 9 \text { desastres. Uma meta-agregação foi } \\
\text { usada para sintetizar os resultados dos estudos e a qualidade foi avaliada } \\
\text { metodicamente com PRISMA e CASP. }\end{array}$ \\
\hline $\begin{array}{c}\text { A2 } \\
\text { BIREME }\end{array}$ & $\begin{array}{l}\text { Identifying Crucial Equipment and Skills } \\
\text { Needed to Evacuate Critically Ill Infants } \\
\text { During Disasters: Using Nursing Expertise to } \\
\text { Guide Training Targets }\end{array}$ & $\begin{array}{l}\text { Gray, Thomas, Burns e } \\
\text { Umoren (2019) }\end{array}$ & $\begin{array}{l}\text { Utilizar a experiência de enfermeiras do departamento } \\
\text { de emergência pediátrica (ED) e na unidade de terapia } \\
\text { intensiva neonatal (UTIN) para determinar as } \\
\text { habilidades de evacuação que as enfermeiras de } \\
\text { cabeceira devem gerenciar de forma independente } \\
\text { durante desastres e para criar uma lista de verificação } \\
\text { de equipamentos essenciais para evacuação de bebês } \\
\text { gravemente enfermos durante desastres. }\end{array}$ & $\begin{array}{l}\text { Seis especialistas participaram desse método iterativo para determinar quais } \\
\text { habilidades e tarefas eram necessárias para uma enfermeira ao lado do leito } \\
\text { evacuar e cuidar com sucesso de um bebê gravemente doente em um desastre. }\end{array}$ \\
\hline $\begin{array}{c}\text { A3 } \\
\text { CINAHL }\end{array}$ & $\begin{array}{l}\text { Congruence } \quad \text { Between } \quad \text { Nurses' } \quad \text { Caring } \\
\text { Behaviors and Patients' Perceptions of Being } \\
\text { Cared for During Disaster Responses }\end{array}$ & Burgos (2019) & $\begin{array}{l}\text { Explorar o conceito de cuidado a partir da perspectiva } \\
\text { de enfermeiras e pacientes que recebem cuidados } \\
\text { durante uma resposta a desastres. }\end{array}$ & $\begin{array}{l}\text { As formulações e estudos dos teóricos sobre cuidados de enfermagem e } \\
\text { desastres são revisados. Uma narrativa do cuidado do autor durante uma } \\
\text { resposta a desastres é descrita, juntamente com um modelo de resposta a } \\
\text { desastres gerado inspirado por teóricos de enfermagem e pontos de vista dos } \\
\text { filósofos sobre o fenômeno do cuidado. }\end{array}$ \\
\hline $\begin{array}{c}\text { A4 } \\
\text { CINAHL }\end{array}$ & $\begin{array}{l}\text { Development And Psychometric Testing Of A } \\
\text { Tool Measuring Nurses' Competence For } \\
\text { Disaster Respons }\end{array}$ & $\begin{array}{l}\text { Marin, Hutton e Witt } \\
(2020)\end{array}$ & $\begin{array}{l}\text { Avaliar as competências dos enfermeiros para resposta } \\
\text { a desastres. }\end{array}$ & $\begin{array}{l}\text { Foram abordados } 20 \text { enfermeiros. } 8 \text { ( } 40 \% \text { ) aceitaram o convite para participar. } \\
\text { Esses } 8 \text { especialistas tinham idades entre } 34 \text { e } 55 \text { anos; e todos trabalhavam no } \\
\text { serviço de atendimento móvel de emergência ou como docente. Dos } 608 \\
\text { enfermeiros que atuam no serviço de atendimento móvel de emergência nos } \\
\text { estados de Santa Catarina e Rio Grande do Sul, } 326 \text { (53\%) aceitaram o } \\
\text { convite para avaliar a viabilidade e confiabilidade do instrumento. }\end{array}$ \\
\hline $\begin{array}{c}\text { A5 } \\
\text { CINAHL }\end{array}$ & $\begin{array}{l}\text { Moments of disaster response in the } \\
\text { emergency department (ED) }\end{array}$ & $\begin{array}{l}\text { Hammad, Arbon, Gebbie } \\
\text { e Hutton (2017) }\end{array}$ & $\begin{array}{l}\text { Descrever cinco momentos distintos de enfermagem no } \\
\begin{array}{lll}\text { departamento de emergência } & \text { (ED) durante uma } \\
\text { resposta a desastres } & & \end{array}\end{array}$ & $\begin{array}{l}\text { Treze enfermeiras da Austrália, Indonésia, Israel, Japão, Quênia, Palestina, } \\
\text { Arábia Saudita e Estados Unidos da América participaram da pesquisa. }\end{array}$ \\
\hline $\begin{array}{c}\text { A6 } \\
\text { CINAHL }\end{array}$ & $\begin{array}{l}\text { Development of disaster nursing education and } \\
\text { training programs in the past } 20 \text { years (2000- } \\
\text { 2019): A systematic review }\end{array}$ & $\begin{array}{l}\text { Loke, Guo e Molassiotis } \\
\text { (2021) }\end{array}$ & $\begin{array}{l}\text { Estabelecer um panorama geral do desenvolvimento } \\
\text { dos programas de educação e treinamento em } \\
\text { enfermagem em desastres nos últimos } 20 \text { anos. }\end{array}$ & $\begin{array}{l}\text { Uma busca sistemática de literatura entre } 2000 \text { a } 2019 \text { foi realizada usando } \\
\text { bancos de dados eletrônicos, incluindo o CINAHL, MEDLINE, PubMed, } \\
\text { Web of Science e Scopus. Um total de } 75 \text { estudos elegíveis foram } \\
\text { identificados a partir de } 3395 \text { artigos potencialmente relevantes }\end{array}$ \\
\hline $\begin{array}{c}\text { A7 } \\
\text { PUBMED }\end{array}$ & $\begin{array}{l}\text { Evolution of } \\
\text { Opportunities for } \\
\text { Nursing } \\
\text { Natural Disaster }\end{array}$ & Sickora et al. (2020) & $\begin{array}{l}\text { Destacar o potencial do socorro em desastres como } \\
\text { uma experiência educacional prática única para } \\
\text { estudantes de enfermagem. }\end{array}$ & $\begin{array}{l}\text { Ao longo de } 6 \text { meses e mais de } 60 \text { viagens, mais de } 200 \text { alunos } \\
\text { (supervisionados por } 12 \text { professores) prestaram cuidados com êxito aos } \\
\text { residentes de Rockport e foram adicionados ao estudo. }\end{array}$ \\
\hline $\begin{array}{c}\text { A8 } \\
\text { PUBMED }\end{array}$ & $\begin{array}{l}\text { Exigências críticas para } \text { a } \text { prática } \text { de } \\
\text { enfermeiros em desastres rurais causados por } \\
\text { inundações }\end{array}$ & Menegat e Witt (2019) & $\begin{array}{l}\text { Identificar as exigências críticas para a prática de } \\
\text { enfermeiros na resposta a desastres hidrológicos na } \\
\text { área rural. }\end{array}$ & $\begin{array}{l}\text { Do total de enfermeiros atuantes }(\mathrm{n}=20), 14 \text { trabalhavam como enfermeiros } \\
\text { da Atenção Primária à Saúde e os demais ocupavam cargos como: } \\
\text { Coordenador de programas de saúde }(\mathrm{n}=3) \text {, responsável pela vigilância em } \\
\text { saúde }(\mathrm{n}=2) \text { e Serviços psicossociais }(\mathrm{n}=1) \text {. Enfermeiros que tinham sido } \\
\text { empregados em seus cargos atuais por até } 5 \text { anos }(\mathrm{n}=7) \text {, de } 6 \text { a } 10 \text { anos }(\mathrm{n}= \\
\text { 6), ou mais de } 11 \text { anos }(\mathrm{n}=7) \text {. A formação incluiu especialização em saúde } \\
\text { pública }(\mathrm{n}=7) \text {, Atenção Primária à Saúde }(\mathrm{n}=5) \text { e atendimento de } \\
\text { emergência }(\mathrm{n}=2) \text {. }\end{array}$ \\
\hline $\begin{array}{c}\text { A9 } \\
\text { PUBMED }\end{array}$ & $\begin{array}{l}\text { Primary health care nurses' competencies in } \\
\text { rural disasters caused by floods }\end{array}$ & Menegat e Witt (2018) & $\begin{array}{l}\text { Identificar as competências dos enfermeiros da atenção } \\
\text { primária à saúde na resposta a desastres hidrológicos } \\
\text { em áreas rurais. }\end{array}$ & $\begin{array}{l}\text { Foram entrevistados } 20 \text { enfermeiros de saúde pública que trabalharam durante } \\
\text { a estação das cheias nos anos de } 2014 \text { e } 2015 \text { em uma área rural no sul do } \\
\text { Brasil. }\end{array}$ \\
\hline
\end{tabular}


Research, Society and Development, v. 11, n. 1, e44811124836, 2022

(CC BY 4.0) | ISSN 2525-3409 | DOI: http://dx.doi.org/10.33448/rsd-v11i1.24836

\begin{tabular}{|c|c|c|c|c|}
\hline $\begin{array}{c}\text { A10 } \\
\text { PUBMED }\end{array}$ & $\begin{array}{l}\text { Mexico City Emergency Nurses Respond After } \\
\text { September } 2017 \text { Earthquake }\end{array}$ & Ortega (2018) & $\begin{array}{l}\text { Avaliar a resposta de enfermeiros de emergência da } \\
\text { cidade do México após o terremoto em setembro de } \\
2017 \text {. }\end{array}$ & $\begin{array}{l}\text { Profissionais de enfermagem atuantes no serviço de emergência e } \\
\text { departamento de emergência da cidade do México. }\end{array}$ \\
\hline $\begin{array}{c}\text { A11 } \\
\text { PUBMED }\end{array}$ & $\begin{array}{l}\text { Disaster preparedness among nurses: a } \\
\text { systematic review of literature }\end{array}$ & Labrague et al. (2018) & $\begin{array}{l}\text { Explorar publicações revisadas por pares que medem a } \\
\text { preparação das enfermeiras para a resposta a desastres }\end{array}$ & $\begin{array}{l}\text { SCOPUS, MEDLINE, PubMed, CINAHL e PsychINFO foram as bases de } \\
\text { dados primárias utilizadas para a pesquisa da literatura. As palavras-chave } \\
\text { usadas nesta revisão foram as seguintes: 'emergência', 'desastre', 'preparação o } \\
\text { para desastres', 'competências em desastres', 'enfermagem em desastres', } \\
\text { 'função em desastres' e 'enfermeiro'. Dezessete (17) artigos foram } \\
\text { selecionados para esta revisão }\end{array}$ \\
\hline
\end{tabular}

Fonte: Artigos pesquisados (2021). 


\section{Discussão}

\section{Competências e habilidades do enfermeiro para atuar frente a situações de desastres naturais e as principais estratégias de enfrentamento}

Os enfermeiros tem papel fundamental frente à ocorrência de desastres naturais, pois são eles que entram em primeiro contado com as vítimas. Neste aspecto, muito se fala em gestão de desastres, onde os profissionais precisam desenvolver habilidades e competências para tornar eficaz o tratamento oferecido aos pacientes, entre elas, merece destaque a resposta ágil, liderança e criatividade, dessa forma a utilização de protocolos podem auxiliar os enfermeiros e minimizar o desperdício de tempo. Vale ressaltar também que os enfermeiros utilizam como estratégias de enfrentamento, a resiliência, comunicação positiva e apoio psicológico, assim, mantendo o autocontrole e atendendo as necessidades dos pacientes (Xue, Shu, Hayter \& Lee, 2020).

Corroborando com a afirmativa, Labrague et al. (2018) afirmam que as evidências científicas apontam como melhor estratégia de enfrentamento, o planejamento, onde as instituições devem seguir protocolos específicos para a atuação frente a ocorrência de desastres naturais e realizar treinamentos com a equipe. Foi observado ainda que, os enfermeiros que já tiveram experiências com atendimentos a pacientes vítimas de desastres tiveram capacidade de reação aumentada. Outro ponto que deve ser citado é a aplicação da tecnologia para auxiliar os atendimentos, melhorar a comunicação e dividir o fluxo de trabalho entre a equipe (Xue et al., 2020).

Mesmo em tempos de crise e situações de urgência e emergência, o cuidado oferecido aos pacientes deve ser humanizado e com foco em minimizar dados físicos e psicológicos. Neste ponto, Burgos (2019) acrescentam que durante o atendimento é necessário ter responsabilidade e honestidade com os clientes, buscando oferecer escuta ativa, quando o mesmo está em condições de verbalizar, oferecer conforto e utilizar o toque para promover segurança e esperança aos clientes.

Diante de um desastre natural, os profissionais podem acabar sem recursos suficientes para prestar cuidados a todos os feridos, portanto, ressalta-se a importância de protocolos, pensamento ágil e criatividade para solucionar os problemas. Na ocorrência de desastres, muitas vezes, é necessário que a população se mobilize para ajudar os profissionais que estão trabalhando para socorrer o maior número possível de pessoas e potencializar o atendimento, onde os enfermeiros podem montar barracas improvisadas para cuidar de pessoas que não estejam gravemente feridas e evitar a superlotação dos hospitais (Ortega, 2018).

No Brasil assim como em outros países, alguns tipos de desastres são mais propensos a acontecer, neste sentido, Menegat e Witt (2018) listam 8 domínios de competências que, os profissionais de enfermagem que atuam na atenção primária devem ter para enfrentar desastres caudados por enchentes, são eles: domínio de gestão e liderança; trabalho de equipe; cuidado de saúde; orientação da comunidade; comunicação; cuidado psicológico; educacional e; vigilância de saúde. Dessa forma, os enfermeiros tem uma base para atender a comunidade e efetivar os cuidados.

Corroborando com o exposto acima, Sickora et al. (2020) salientam a importância da atenção primária frente ao enfrentamento de desastres naturais, onde a prevenção primária, secundária e a realização de treinamentos se faz presente juntamente com a prestação de cuidados de emergências. Os profissionais precisam estar preparados ainda, para possíveis evacuações, onde precisariam transportar os pacientes em segurança e evitar possíveis agravos a saúde dos clientes (Gray et al., 2019).

Neste segmento, é importante ressaltar que algumas áreas são frequentemente afetadas por desastres naturais, como por exemplos, áreas de risco para ocorrência de enchentes, neste aspecto, a atuação do enfermeiro é diferente da sua atuação frente a um desastre natural totalmente inesperado. Neste caso, os profissionais focam em prevenção e tem protocolos bem definidos para orientar suas ações, visando diminuir as perdas da comunidade (Menegat \& Witt, 2019).

Ainda sobre a gestão dos desastres, Loke et al. (2021) destacam a necessidade da criação de programas de educação e 
treinamento de enfermagem de desastres, e treinamentos de aprendizagem colaborativa. Corroborando com o exposto, Hammad et al. (2017) enaltece que a determinação dos enfermeiros para atuar frente a ocorrência de desastres é fonte de apoio para todos os profissionais e elenca que as ações da equipe de enfermagem são baseadas em 5 momentos, sendo eles: Notificação, espera, chegada do paciente, cuidados com o paciente e reflexão, onde os profissionais precisam ter agilidade e autocontrole para realizar suas funções.

Por fim, deve-se mencionar que a atuação da enfermagem frente à ocorrência de desastres naturais está ligada a quatro áreas de competências especificas que estão correlacionadas, são elas: competências de mitigação, competências de preparação, competências de resposta e competências de reabilitação, onde a união das competências é fundamental para prestar uma assistência de qualidade aos pacientes e garantir uma maior sobrevida dos mesmos (Marin et al., 2020).

\section{Principais riscos que podem afetar a saúde dos enfermeiros que atuam nos desastres naturais}

Um dos riscos mais citados que podem comprometer a saúde dos profissionais de enfermagem durante sua atuação frente a um desastre natural é a falta de preparo suficiente. De acordo com Loke et al. (2021), o treinamento e educação em enfermagem de desastres é de suma importância para todos os níveis, tanto estudantes como profissionais já formados.

Estudo feito no Texas implementou um curso preparatório em uma Universidade para ocorrência de desastres e demonstrou que após ele os alunos puderam vivenciar o papel da equipe na ocorrência de desastres naturais, além disso forneceu ensinamentos como resposta rápida, avaliação de necessidades e mapeamento (Sickora et al., 2020). Em concordância, Gray et al. (2019) afira que atividades preparatórias para esse tipo de situação trazem habilidades essenciais que garantem aos enfermeiros gerenciar problemas e buscar soluções durante uma emergência.

A falta de recursos e falha de equipamento também são listadas como um risco para os trabalhadores, uma vez que dificultam, por exemplo, as operações de resgate, aumentam a carga de trabalho e reduzem a eficiência clínica do trabalho (Xue et al., 2020).

Outro risco é a escassez de diretrizes que apoiem as práticas dos profissionais. Estudos dissertam que a implantação de protocolos aumenta o nível de respostas em emergências e agiliza o trabalho, sendo extremamente necessários no atendimento a desastres (Menegat \& Witt, 2018; Menegat \& Witt, 2019). Corroborando, Xue et al. (2020) relatam que protocolos e diretrizes são úteis tanto antes, durante como após o socorro, pois eles servem para ajudar gestores, refinar elementos-chave no campo e avaliar sistemas de alerta precoce.

\section{Grau de preparação dos profissionais de saúde para atuar frente a situações de desastres}

Apesar do uso de diferentes escalas para verificar a preparação dos enfermeiros frente a desastres, um achado comum entre os estudos é que os profissionais ainda não estão adequadamente preparados para enfrentar tais situações, entretanto, com a maior ocorrência de desastres naturais nos últimos anos já se percebe mudanças e atualizações quanto a essa preparação.

Estudo feito na Austrália demonstrou, por meio de entrevistas, que os enfermeiros não estão devidamente treinados para responder a desastres, muitos deles reagem de forma inapropriada a notificação de um desastre, questionando suas habilidades, torcendo para que as lesões dos pacientes sejam semelhantes aos vistos no dia a dia do trabalho (Hammad et al., 2017). Corroborando, o estudo de Ortega (2018) descreve a situação desencadeada por um terremoto que atingiu o México em 2017, no qual nem os hospitais e nem profissionais de saúde tinham a preparação adequada para enfrentamento de desastres naturais, o que causou caos e dificultou o atendimento aos pacientes.

O desempenho de enfermeiros em todas as situações enfrentadas por eles depende da sua preparação, e em situações de desastre não é diferente, contudo, essas dependem ainda da implementação de diretrizes, protocolos, e acordos regionais para que o trabalho aconteça de forma resiliente (Xue et al., 2020). 


\section{Considerações Finais}

As evidências científicas demonstram que o enfermeiro tem um papel fundamental diante da ocorrência de desastres naturais, sendo o primeiro a prestar atendimento a vítima através do seu conhecimento técnico-cientifico e prático de urgência e emergência, utilizando habilidades como gestão e trabalho em equipe, criando estratégias essências para um atendimento adequado. Além disso, as tecnologias também podem ser aliadas bastante eficientes nesse atendimento inicial, contribuindo para a sobrevida do paciente.

Diante dos problemas encontrados, fica claro que assistência prestada aos pacientes vítimas de desastres naturais, deve ser focada em aspectos físicos e psicológicos. Nesse caso, a enfermagem deve entrar em ação lidando diretamente com o sujeito e prestando esclarecimentos acerca do procedimento realizado por ele, além de realizar escuta ativa, quando o paciente tem condições e deseja verbalizar, oferecer conforto e utilizar o toque como fonte de segurança aos clientes.

Já que a enfermagem exerce importante papel no atendimento de pacientes em situações de desastres naturais, e é uma ligação entre o sujeito e os demais profissionais de saúde, os enfermeiros devem avaliar a qualidade da assistência prestada, encaminhando o paciente a outros profissionais (psicólogo, fisioterapeuta, nutricionista, etc.) sempre que necessário, e monitorando o grau de conhecimento do indivíduo e da família acerca do seu tratamento tanto pré-hospitalar quanto intrahospitalar.

Como limitações do estudo tem-se o fato da escassez de literatura sobre a atuação da enfermagem em situações de desastres no período pré-hospitalar e demais momentos. Além disso, as evidências científicas não estão muito atualizadas, onde houve dificuldade para utilizar novos autores.

Por fim, o estudo possibilitou expandir os conhecimentos acerca dos tipos de desastres naturais, os fatores relacionados a eles e a atuação dos profissionais de enfermagem para o atendimento de vítimas de desastres. Dessa forma, considera-se que novas pesquisas devem ser realizadas nesta linha de investigação, a fim de provar a importância de mais pesquisas relacionadas ao tema, a realização de treinamentos periódicos e a utilização de protocolos de atendimento para auxiliar os enfermeiros.

\section{Referências}

Bork, A. M. T. (2011). Enfermagem baseada em evidências. Rio de Janeiro: Guanabara Koogan.

Burgos, G. (2019). Congruence between nurses' caring behaviors and patients' perceptions of being cared for during disaster responses. International Journal for Human Caring, 23 (1): 80-91.

Cohen, G. H., Tamrakar, S., Lowe, S., Sampson, L., Ettman, C., Kilpatrick, D., \& Galea, S. (2019). Improved social services and the burden of post-traumatic stress disorder among economically vulnerable people after a natural disaster: a modelling study. Lancet Planet Health, 3(2).

Gray, M. M., Thomas, A. A., Burns, B. \& Umoren, R. A. (2019). Identifying crucial equipment and skills needed to evacuate critically ill infants during disasters: using nursing expertise to guide training targets. Prehospital and Disaster Medicine, 34 (4): $370-375$.

Guha-Sapir, D., Hoyois, P. \& Below, R. (2015). Annual Disaster Statistical Review 2014: The Numbers and Trends. Brussels: CRED, 2015.

Hammad, K. S., Arbon, P., Gebbie, K. \& Hutton, A. (2017). Moments of disaster response in the emergency department (ED). Australas Emerg Nurs J, 20 (4): $181-185$.

Hamatate, Y., Sasaki, Y. \& Miura, H. (2019). Problemas reais e problemas de contramedidas de desastres para empresas em Chiyoda-ku, Tóquio. Revista de Higiene Industrial, 61 (3).

Jillson, I. A., Clarke, M., Allen, C., Waller, S., Koehlmoos, T., Mumford, W., \& Trant, A. (2019). Improving the science and evidence base of disaster response: a policy research study. BMC Health Serv Res, 19 (274).

Labrague, L. J., Hammad, K., Gloe, D. S., McEnroe-Petitte, D. M., Fronda, D. C., Obeidat, A. A., \& Mirafuentes, E. C. (2018). Disaster preparedness among nurses: a systematic review of literature. Int Nurs Rev., 65(1):41-53.

Loke, A.Y., Guo C. \& Molassiotis, A. (2021). Development of disaster nursing education and training programs in the past 20 years (2000-2019): A systematic review. Nurse Educ Today, 99:104809. 
Research, Society and Development, v. 11, n. 1, e44811124836, 2022

(CC BY 4.0) | ISSN 2525-3409 | DOI: http://dx.doi.org/10.33448/rsd-v11i1.24836

Marin, S. M., Hutton, A. \& Witt, R. R. (2020). Development and Psychometric Testing of a Tool Measuring Nurses' Competence for Disaster Response. $J$ Emerg Nurs. 46(5):623-632.

Menegat, R. P. \& Witt, R. R. (2019). Critical Requirements for Nursing Practice in Rural Disasters Caused by Floods. Revista Brasileira de Enfermagem, 72 (3): 687-691.

Menegat, R. P. \& Witt, R. R. (2018). Primary health care nurses' competencies in rural disasters caused by floods. Rural and Remote Health,18 (3).

Moos, C., Bebi, P., Schwarz, M., Stoffel, M., Sudmeier-Rieux, K., \& Dorren, L. (2018). Redução do risco de desastres com base no ecossistema nas montanhas. Earth-Science Reviews, 177: 497-513.

Oliveira, F. B. M., Acrychyld, C. A. L. C., Alves, D. L., França, J. F., Macedo, M. S. \& Santos, R. D. (2016). Relação entre a sobrecarga de trabalho e erros de administração de medicação na assistência hospitalar. Revista Ciências \& Saberes. 2(2): 325-334.

Ortega, J. G. (2018). Mexico City Emergency Nurses Respond After September 2017 Earthquake. J Emerg Nurs. 44(2):200-202.

Sickora, C., Salt, R. J., Page, T. S., Lee, W., Cantu, A. G., Lee, M., \& Byrd, D. (2020). Evolution of Experiential Learning Opportunities for Nursing Students After Natural Disaster. J Nurs Educ. 59(1):30-33.

Sousa, L. M. M., Marques-Vieira, C. M. A., Severino, S. S. P. \& Antunes, A. V. (2017). Metodologia de Revisão Integrativa da Literatura em Enfermagem. Revista Investigação Enfermagem, 2(21): 17-26.

Souza, M. T., Silva, M. D. \& Carvalho, R. (2010). Revisão integrativa: o que é e como fazer. Einstein, 8: 102-6.

Xue, C. L., Shu, Y. S., Hayter, M. \& Lee, A. (2020). Experiences of nurses involved in natural disaster relief: A meta-synthesis of qualitative literature. J Clin Nurs. 29(23-24):4514-4531.

Zhouri, A., Valencio, N., Oliveira, R., Zucarelli, M., Laschefski, K. \& Santos, A. F. (2016). O desastre da Samarco e a política das afetações: classificações e ações que produzem o sofrimento social. Cienc. Cult., 68 (3): 36-40. 